\title{
Time-of-flight SIMS as a novel approach to unlocking the hypoxic properties of cancer
}

\author{
Emily G Armitage, ${ }^{a}$ Helen L Kotze, ${ }^{a}$ John S Fletcher, ${ }^{b}$ Alex Henderson, ${ }^{b}$ \\ Kaye J Williams, ${ }^{c}$ Nicholas P Lockyer ${ }^{d *}$ and John C Vickerman ${ }^{b}$
}

\begin{abstract}
It is known that hypoxia-inducible factor 1 (HIF-1) activity results in the coordinated up-regulation of a large number of proteins that facilitate cell survival in tumours; however, the effect of HIF-1 on cancer metabolism is less well characterised. With knowledge of the specific effect of HIF-1 on cancer metabolism, biomarkers could be identified for which new drugs could be targeted. Time-of-flight secondary ion mass spectrometry (ToF-SIMS) offers the potential to analyse intact cells in situ and has a mass spectral coverage that is applicable to metabolic profiling. It has been used to analyse the effects of HIF-1 on multicellular tumour models. Multicellular tumour spheroids (MTSs) have been cultured from human colon carcinoma cells with and without the expression of HIF-1, and the surface of the cross sections of each MTS has been analysed. Because metabolic profiling is an emerging field in ToF-SIMS, there is a requirement to determine which metabolites can be detected using this technique and which of those can be identified in complex mixtures within biological samples. For this, a selection of metabolites have been analysed, and the ToF-SIMS standard spectra acquired have been used to localise metabolites in MTS sections. The comparison of metabolic profiles of MTSs with and without the expression of HIF-1 has elucidated potential biomarkers for tumour survival in hypoxia, some of which may be HIF-1 regulated. Copyright $\odot 2012$ John Wiley \& Sons, Ltd.
\end{abstract}

Keywords: metabolomics; ToF-SIMS; cancer; hypoxia; HIF; PCA

\section{Introduction}

\section{Hypoxia}

When cells are growing in a region that is greater than $100 \mu \mathrm{m}$ from an oxygen supply, they are described as hypoxic ${ }^{[1]}$ (living in a low oxygen environment). It is known that solid tumours are hypoxic and that they must promote their own survival by responding to low oxygen. Many responses of tumour cells to hypoxia are orchestrated by the activation of hypoxia-inducible factors (HIFs). ${ }^{[2]}$ HIFs are transcription factors that are controlled at the level of post translational regulation and are responsible for regulating the expression of more than 100 target genes. ${ }^{[3]}$ HIF-1 is one of the key players in the HIF family and consists of two subunits: HIF- $1 \alpha$ and HIF-1 $\beta$ that can exist independently in well-oxygenated cells or as an active complex in hypoxic cells. ${ }^{[4]}$ Deficiencies or blocking of either subunit stops the formation of the active heterodimer; thus, the hypoxic response can no longer be HIF-1 mediated. In this study, multicellular tumour spheroids (MTSs) have been cultured from human colon HCT116 cells expressing a dominant-negative form of HIF- $1 \alpha$ (HCT116 DN), thus preventing HIF-1 activity, and have been compared with MTSs cultured from wild type cells expressing HIF-1 normally (HCT116 WT).

\section{ToF-SIMS and metabolic profiling}

Metabolic profiling is an extensive but qualitative analysis of all detectable metabolites in a biological sample. It is possible to observe metabolic changes, including the presence, absence or relative changes in concentrations across the entire metabolic network. The data can be used to make relative comparisons between biological samples that are subjected to different environmental conditions such as level of oxygen or to determine the effect of a perturbation such as HIF-1 deficiency. Time-of-flight secondary ion mass spectrometry (ToF-SIMS) has the potential to provide a new tool in metabolic profiling for the detection and imaging of metabolites in cellular or tissue samples, thus enabling in situ profiling. This puts ToF-SIMS at a distinct advantage over other techniques commonly used in metabolic profiling such as chromatography coupled mass spectrometry or nuclear magnetic resonance. Imaging mass spectrometry is currently of increasing importance in the biosciences and is receiving a significant amount of attention in the mass spectrometric community. ${ }^{[5]}$ It has been successful in the imaging of biological samples from cells ${ }^{[6]}$ to tissue. ${ }^{[7,8]}$ Imaging ToF-SIMS has been used in this study as the platform for the metabolic profiling of MTSs cultured from HCT116 WT cells or HCT116 DN cells to investigate the phenotype of each sample. To aid interpretation of the MTS data, the reference ToF-SIMS spectra of selected metabolites were first acquired.

\footnotetext{
* Correspondence to: Nicholas Lockyer, School of Chemistry, University of Manchester, Oxford Road, Manchester M13 9PL, UK.

E-mail: Nick.Lockyer@manchester.ac.uk

a Doctoral Training Centre for Integrative Systems Biology, School of Chemical Engineering and Analytical Science, Manchester Interdisciplinary Biocentre, University of Manchester, Manchester M1 7DN, UK

b Surface Analysis Research Centre, School of Chemical Engineering and Analytical Science, Manchester Interdisciplinary Biocentre, University of Manchester, Manchester M1 7DN, UK

c School of Pharmacy and Pharmaceutical Sciences, University of Manchester, Oxford Road, Manchester M13 9PT, UK

d School of Chemistry, University of Manchester, Oxford Road, Manchester M13 SPL, UK
} 


\section{Experimental}

\section{Metabolite standards}

Standard ToF-SIMS spectra were acquired for metabolites (SigmaAldrich, Dorset, UK) analysed on a Bio ToF-SIMS instrument described previously by Braun et al. ${ }^{[9]}$ and briefly in the following sections. The instrument was equipped with a Wien filtered 20-kV $\mathrm{C}_{60}{ }^{+}$ion gun (lonoptika Ltd, Southampton, UK) mounted at an angle of $50^{\circ}$. For analysis, the raster field covered a $250 \times 250-\mu \mathrm{m}^{2}$ area of each sample, and the primary ion dose was maintained at $1 \times 10^{11}$ ions $\mathrm{cm}^{-2}$. Standards were prepared in HPLC-grade $\mathrm{H}_{2} \mathrm{O}$ (Sigma-Aldrich) to a volume of $1 \mathrm{mM}$ and were spotted onto silicon substrates (Advent RM, Oxford, UK) at volumes of $2 \mu \mathrm{l}$.

\section{Preparation of MTS sections}

HCT116 WT and DN cells were obtained from the University of Manchester cell stocks, and a high throughput method for the culturing of MTSs was designed in house. This method involved seeding approximately $3 \times 10^{6}$ cells in a T75 flask (Falcon, Runcorn, UK) containing $30 \mathrm{ml} \mathrm{RPMI} \mathrm{medium} \mathrm{(Gibco} \mathrm{BRL,} \mathrm{Paisley,} \mathrm{UK} \mathrm{)} \mathrm{supple-}$ mented with $10 \%$ foetal calf serum (Biosera, East Sussex, UK) and 2 $\mathrm{mM}$ L-glutamine (Sigma-Aldrich) in a $95 \%$ air $/ 5 \% \mathrm{CO}_{2}$ environment. Flasks were shaken constantly (Bibby Scientific Ltd, Staffordshire, UK) for 3 weeks within an incubator. Subsequently, MTSs were set directly into $1.5 \%$ gelatine heated to $60^{\circ} \mathrm{C}$ and were then immediately snap frozen in liquid nitrogen and stored at $-80^{\circ} \mathrm{C}$. MTSs were sectioned at $10 \mu \mathrm{m}$ using a cryostat microtome (Bright, Starlet 2212; Instrument Company Ltd, Huntingdon, UK) operated at approximately $-20^{\circ} \mathrm{C}$ directly before analysis to minimise degradation from the thawing process. The sections were subsequently thaw mounted onto pre cooled silicon substrates. MTS sections were washed three times in $0.15 \mathrm{M}$ ammonium formate each for $1 \mathrm{~min}$ and dried on a WS-400-6NPP/LITE spin caster (Laurell technologies, Pennsylvania, USA) operated at 800 rpm for $10 \mathrm{~min}$, followed by vacuum desiccation before ToF-SIMS analysis. The embedding medium used to attach the sample to the disk was optimal cutting temperature (OCT) polymer. This is commonly used in histology to stabilise the tissue and provides a smooth cutting surface; however, it poses problems for samples to be analysed using imaging mass spectrometry as it has been shown to suppresses ion formation. ${ }^{[10]}$ Therefore, for this purpose, the amount of OCT used was kept to a minimum to ensure no OCT contaminates the cross section to be analysed.

\section{ToF-SIMS imaging analysis of MTS sections}

MTS sections were analysed using the J105 3D Chemical Imager (lonoptika Ltd) described previously by Fletcher et al. $^{[11]}$ and briefly in the following sections. The instrument has been developed by Vickerman and coworkers at the University of Manchester and uses a continuous primary ion beam to generate a continuous stream of secondary ions that are sampled by a buncher, which creates a tight packet of ions at the entrance to a harmonic field reflectron. A $40-\mathrm{kV} \mathrm{C}_{60}{ }^{+}$primary ion gun was used, mounted at an angle of $45^{\circ}$. For analysis, the raster field covered an area of $1000 \times 1000 \mu \mathrm{m}^{2}$, and a primary ion dose of $2.45 \times 10^{13}$ ions $\mathrm{cm}^{-2}$ was used. Each image was generated as a $256 \times 256$ pixel square where the spot size was $5 \mu \mathrm{m}$. Because of file size limitations; the data were down sampled during acquisition to 0.05 -Da bins.

\section{Data processing}

Multivariate analysis is widely used in ToF-SIMS for reducing the dimensionality of multivariate data while preserving most of the variance. $^{[12]}$ In particular, principal components analysis (PCA) reduces the dimensionality by identifying combinations of data variables that account for more of the variation in the total data set than any other individual data variable, thus creating new combinations of variables termed principal components. ${ }^{[13]}$ To improve image contrast, image PCA can be conducted for a whole image or a region of interest within the image. ${ }^{[13]}$ Image PCA was performed on one MTS from each total ion image using in-house developed routines for MATLAB R2009a version 7.8 software that use the Image Processing Toolbox and Statistics Toolbox (Math Works, Inc, Massachusetts, USA). The principal component that seemed to account for oxygen variation was selected for each MTS, and the metabolite standard spectra were used to identify some of the anticorrelation of features in the loadings. From this, it was determined whether or not each anticorrelation may be due to a HIF-1 regulated response to hypoxia.

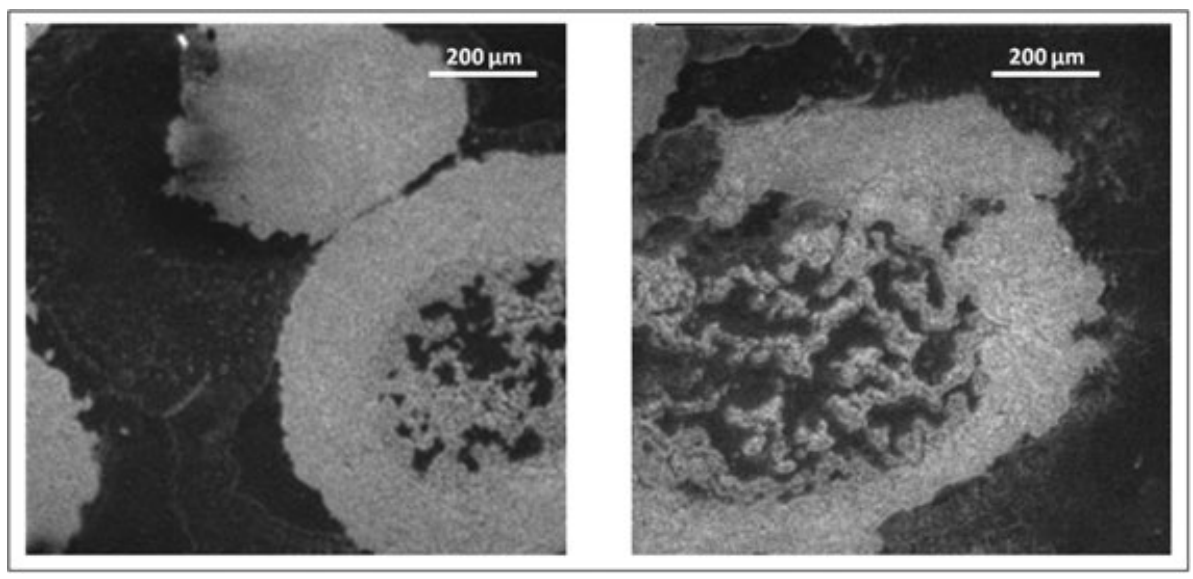

Figure 1. Total ion images from the analysis of HCT116 WT (left) and DN (right) MTSs. Necrotic regions are seen in the centre of the spheroids where cellular structure has been disrupted. Images were acquired in a positive-ion mode using $40 \mathrm{keV} \mathrm{C}_{60}{ }^{+}$. 

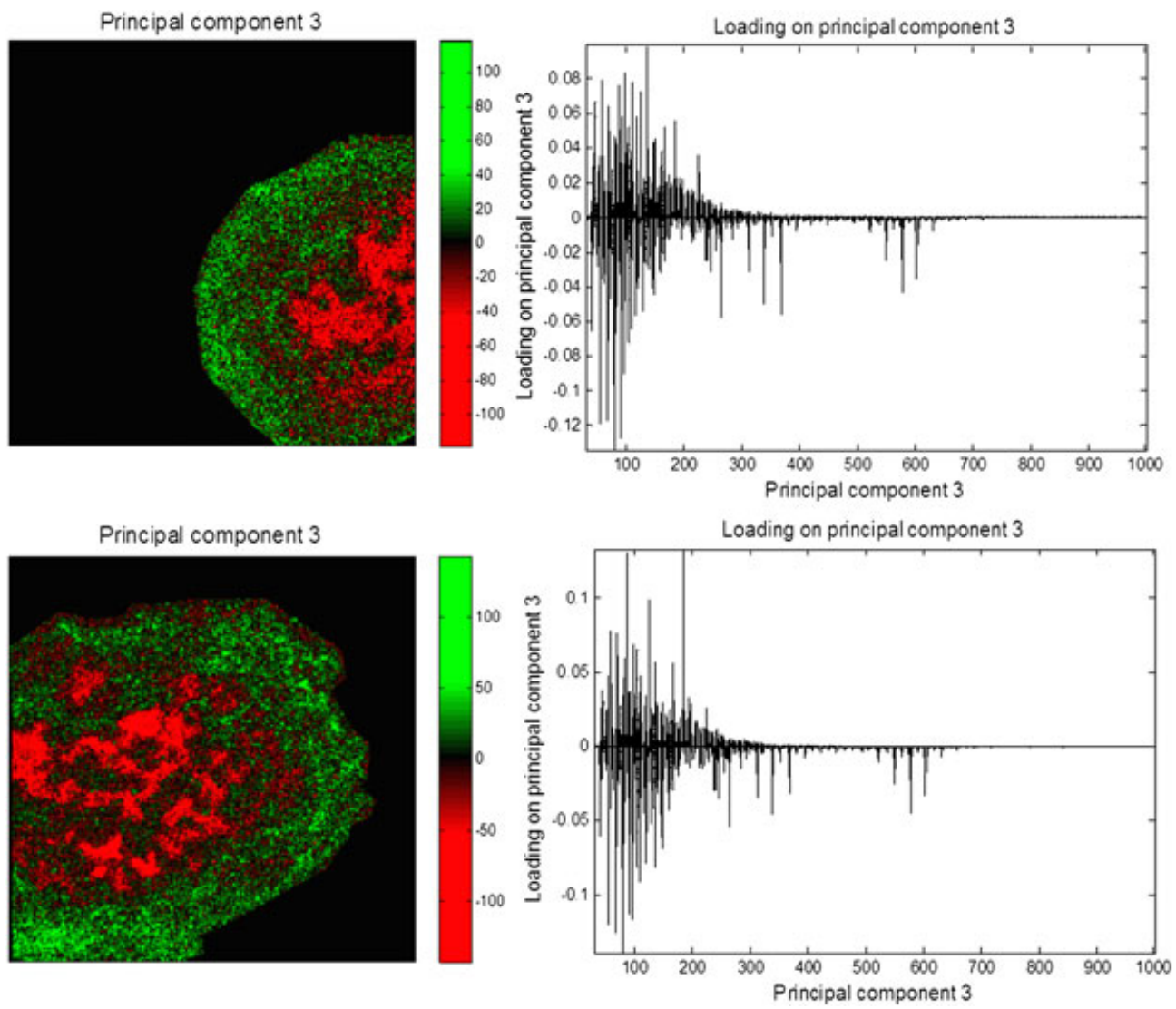

Figure 2. Scores and loadings from image PCA on one MTS from each total ion image from the analysis of HCT116 WT (top) and DN (bottom).
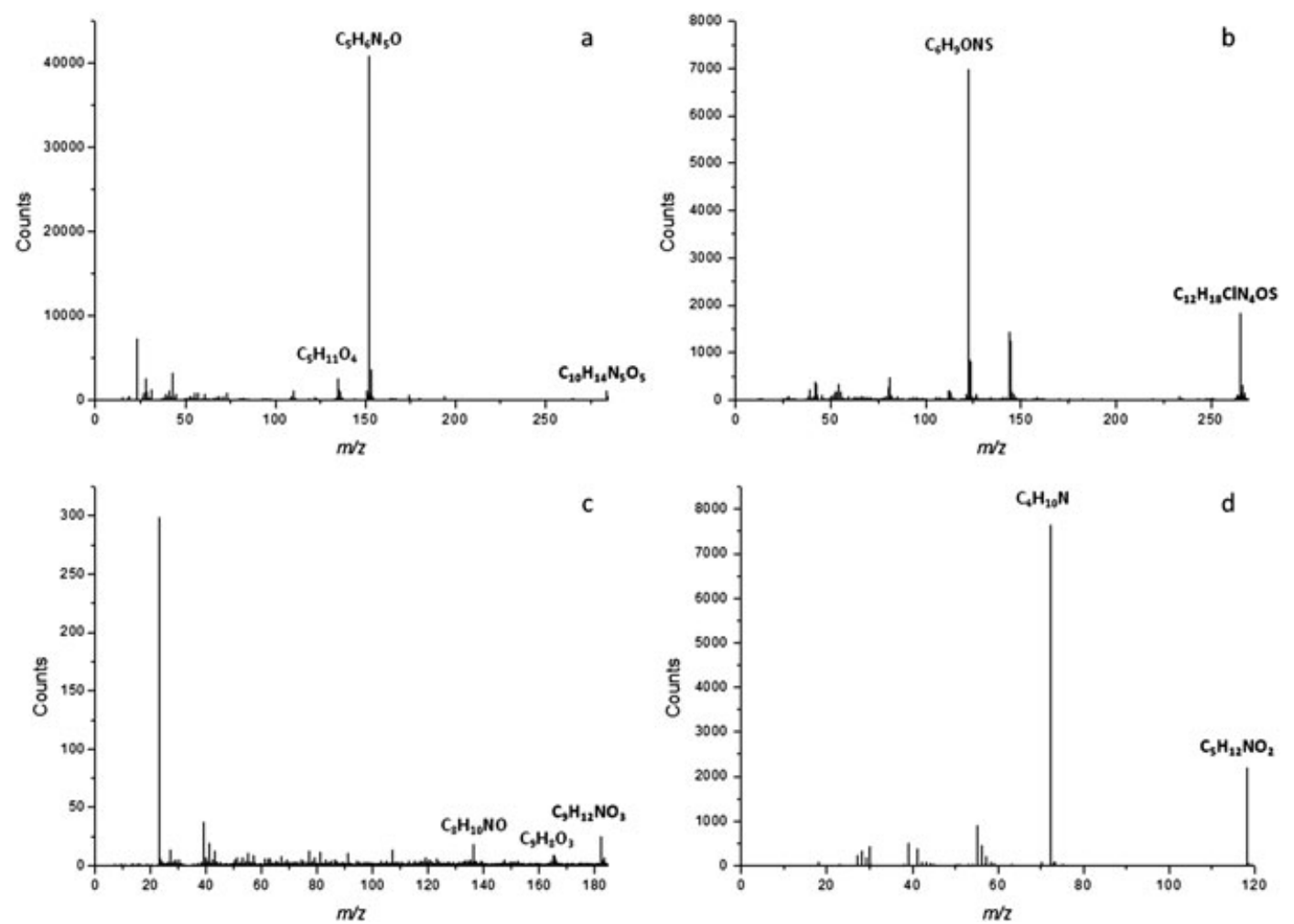

Figure 3. Standard spectra for (a) guanosine, (b) thiamine, (c) tyrosine and (d) valine analysed at a concentration of $1 \mathrm{mM}$ using ToF-SIMS with a 20 keV $\mathrm{C}_{60}{ }^{+}$primary ion beam in a positive-ion mode. For each standard analysed, the formula of $[\mathrm{M}+\mathrm{H}]^{+}$is indicated in bold and suggested chemical formulae of major fragments are labelled. 


\section{Results and discussion}

Sections of MTSs cultured from HCT116 WT or DN cells have been analysed in a positive-ion mode using $\mathrm{C}_{60}{ }^{+}$ToF-SIMS. Figure 1 shows the total ion images from each analysis. Image PCA has been used to assess the variation in a single MTS from each image. The scores and the loadings plots for the principal component that accounts for variation due to the oxygen gradient in image PCA are shown in Fig. 2, where positive scores are green and negative scores are red in the scores plot, and the loadings are plotted against the $m / z$ values. For both the HCT116 WT MTS and the HCT116 DN MTS, image PCA revealed variation where the chemical anticorrelation between hypoxic cells in the centre of the MTS and more oxygenated cells surrounding the centre was observed. It has been highlighted previously that the metabolic phenotype of HIF-1deficient cells differs from WT cells when exposed to low oxygen ${ }^{[14]}$; hence, it is likely that these chemical differences are metabolites.

Metabolites have been analysed in a positive-ion mode using $\mathrm{C}_{60}{ }^{+}$ToF-SIMS, and the standard spectra have been used to identify peaks in image PCA loadings that account for the separation in each image. In both MTSs, valine is correlated with tyrosine, whereas in the HCT116 WT MTS, thiamine is anticorrelated with guanosine. This anticorrelation was not observed in the HCT116 DN MTS, suggesting that one or more of the pathways involving these metabolites may be downstream targets of HIF-1 and furthermore highlights the action of HIF-1 at the amino acid level. The anticorrelation between guanosine and thiamine in the HCT116 WT MTS provides evidence that suggests an HIF-1-mediated response to hypoxia at the level of DNA. Figure 3 shows the standard spectra for the four metabolites highlighted in the image PCA loadings. In each, the chemical formula for the quasi-molecular ion is shown, and probable chemical formulae have been assigned to the most intense fragments. Fragments have been compared with mass spectra acquired by liquid chromatography electrospray ionisation mass spectrometry using MassBank: a public repository for sharing mass spectral data for life sciences. ${ }^{[15]}$ For all four metabolites, the ToF-SIMS fragments and the liquid chromatography electrospray ionisation mass spectrometry fragments were well matched; hence, it may be possible to use this repository to identify further unknown features of ToF-SIMS spectra.

\section{Conclusion}

ToF-SIMS has been used to analyse the cross sections of MTSs cultured from wild-type human colon cancer cells (HCT116 WT) and cells of the same origin that express a dominant-negative form of the HIF- $1 \alpha$ subunit (HCT116 DN). The image PCA of one MTS from each cross section has revealed anticorrelated metabolites that are suggestive of hypoxic targets, some of which may be involved in one or more pathways that are downstream targets of HIF-1. This work has demonstrated the potential use of ToF-SIMS in metabolic studies of tumour models. This approach has been extended to tissue samples and will be reported in a future publication.

\section{Acknowledgements}

This work has been funded by the Biotechnology and Biological Sciences Research Council and the Engineering and Physical Sciences Research Council.

\section{References}

[1] VA Carroll, M Ashcroft, Expert Rev. Mol. Med. 2005, 7, 1.

[2] K Ruan, G Song, GL Ouyang, J. Cell. Biochem. 2009, 107, 1053.

[3] G. L. Semenza, Curr. Opin. Genet. Dev., 20, 51.

[4] GL Wang, BH Jiang, EA Rue, GL Semenza, Proc. Natl. Acad. Sci. U. S. A. 1995, 92, 5510.

[5] RMA Heeren, DF Smith, J Stauber, B Kükrer-Kaletas, L MacAleese, J. Am. Soc. Mass Spectrom. 2009, 20, 1006

[6] JS Fletcher, Analyst 2009, 134, 2204.

[7] D Touboul, A Brunelle, F Halgand, S De La Porte, O Laprevote, J. Lipid Res. 2005, 46, 1388.

[8] D Debois, M-P Bralet, FO Le Naour, A Brunelle, O Laprévote, Anal. Chem. 2009, 81, 2823.

[9] RM Braun, P Blenkinsopp, SJ Mullock, C Corlett, KF Willey, JC Vickerman, N Winograd, Rapid Commun. Mass Spectrom. 1998, 12, 1246.

[10] SA Schwartz, ML Reyzer, RM Caprioli, J. Mass Spectrom. 2003, 38, 699.

[11] JS Fletcher, S Rabbani, A Henderson, P Blenkinsopp, SP Thompson, NP Lockyer, JC Vickerman, Anal. Chem. 2008, 80, 9058.

[12] JS Fletcher, A Henderson, RM Jarvis, NP Lockyer, JC Vickerman, R Goodacre, Appl. Surf. Sci. 2006, 252, 6869.

[13] BJ Tyler, G Rayal, DG Castner, Biomaterials 2007, 28, 2412.

[14] H Troy, YL Chung, M Mayr, L Ly, K Williams, I Stratford, A Harris, J Griffiths, M Stubbs, Metabolomics 2005, 1, 293.

[15] H Horai, M Arita, S Kanaya, Y Nihei, T Ikeda, K Suwa, Y Ojima, K Tanaka, S Tanaka, K Aoshima, Y Oda, Y Kakazu, M Kusano, T Tohge, F Matsuda, Y Sawada, MY Hirai, H Nakanishi, K Ikeda, N Akimoto, T Maoka, H Takahashi, T Ara, N Sakurai, H Suzuki, D Shibata, S Neumann, T lida, K Tanaka, K Funatsu, F Matsuura, T Soga, R Taguchi, K Saito, T Nishioka, J. Mass Spectrom. 45, 703. 